\title{
A historical perspective of algorithmic lateral inhibition and accumulative computation in computer vision ${ }^{\text {is }}$
}

\author{
Antonio Fernández-Caballero a,b,*, María T. López ${ }^{\mathrm{a}, \mathrm{b}}$, Enrique J. Carmona ${ }^{\mathrm{c}}$, Ana E. Delgado ${ }^{\mathrm{c}}$ \\ a Instituto de Investigación en Informática de Albacete (I3A), 02071 Albacete, Spain \\ b Universidad de Castilla-La Mancha, Departamento de Sistemas Informáticos, 02071 Albacete, Spain \\ c Universidad Nacional de Educación a Distancia, Departamento de Inteligencia Artificial, 28040 Madrid, Spain
}

\section{A R T I C L E I N F O}

Available online 27 October 2010

Keywords:

Algorithmic lateral inhibition

Accumulative computation

Neuro-inspired computer vision

\begin{abstract}
A B S T R A C T
Certainly, one of the prominent ideas of Professor José Mira was that it is absolutely mandatory to specify the mechanisms and/or processes underlying each task and inference mentioned in an architecture in order to make operational that architecture. The conjecture of the last fifteen years of joint research has been that any bottom-up organization may be made operational using two biologically inspired methods called "algorithmic lateral inhibition", a generalization of lateral inhibition anatomical circuits, and "accumulative computation", a working memory related to the temporal evolution of the membrane potential. This paper is dedicated to the computational formulation of both methods. Finally, all of the works of our group related to this methodological approximation are mentioned and summarized, showing that all of them support the validity of this approximation.
\end{abstract}

(c) 2010 Elsevier B.V. All rights reserved.

\section{Introduction}

One of the most important problems in Artificial Intelligence (AI) and in Computational Neuroscience $(\mathrm{CN})$ is to find effective calculation procedures that enable connecting the analytic models of the behavior of individual neurons, typical of the Neurodynamics $[3,2]$, with the formulations in natural language of the concepts and inferences associated to the high-level cognitive processes $[30,39,35]$. Four years ago, the research community has celebrated the fiftieth anniversary of $\mathrm{AI}[16]$ and it is evident that there is still no general and satisfactory solution to this problem, as clearly stated by Professor José Mira in one of his last papers [36]. The reasons for this lack of links between the models belonging to Physics and AI reside in the intrinsic complexity of the cognitive processes and in the lack of adequate methodological approaches.

The search for architectures of the cognition (the "logic of the mind") is a long-haul task with very limited results that has roots in the ancient Greece. Since then the task has essentially been of interest to philosophers, neurophysiologists, psychologists, mathematicians and, more recently, professionals of the field of

\footnotetext{
This article is dedicated to the memory of Professor José Mira, a great researcher, a wise man, a loving husband, and a close friend; but who sadly passed away on August 13, 2008.

* Corresponding author at: Universidad de Castilla-La Mancha, Departamento de Sistemas Informáticos, 02071 Albacete, Spain. Tel.: + 34967599200 ; fax: + 34967599224 .

E-mail address: caballer@dsi.uclm.es (A. Fernández-Caballero).
}

computation in general and of $\mathrm{AI}$ in particular. We are in front of a partial, fragmented and non-structured knowledge and look for an abstract structure that allows us to order adequately these pieces of knowledge. The architecture needs organization and structure, easy indexation and search and, finally, efficient use of this knowledge in inference and reasoning.

In the robotics field the name architecture is kept explicitly and such terms as "reactive", "situated" or "representational" are used to specify the organization of the software and hardware of an autonomous robot. In AI these organizational approaches are usually called paradigms and it is again distinguished between connectionist, situated, symbolic or representational and hybrid. In this work our architecture of hybrid character, which combines a bottom-up part of connectionist type with a top-down one of symbolic type is presented. To fix ideas we focus on the visual path but we also give out the conjecture of the potential validity in other sensory modalities and in the understanding and synthesis of other tasks in which reactive components are combined with others of intentional nature.

The ideas underlying the work are: (1) In order to theorize and to solve problems that go beyond the retinoscopic projection step it is necessary to propose neurophysiologically plausible synthetic architectures (knowledge models), but without the precision by which the more peripheral structures are known. (2) It is not sufficient to model and interpret the neuronal function at physical (physiological) level of registrations of slow potentials or spikes trains. It is necessary to re-formulate the neuronal mechanisms at symbolic level in terms of inferential rules and frames, closer to the natural language used by an external observer when describing the 
visual processes, or when performing psychophysical experiments $[21,20]$.

The rest of the paper is structured as follows. In Section 2 the two neuronal processes, algorithmic lateral inhibition (Section 2.1) and accumulative computation (Section 2.2), are described in extensive. Then, in Section 3 the use of both processes are described in computer vision applications. Lastly, we offer some conclusions.

\section{Description of the two neural processes}

In order to make operational any architecture it is necessary to specify the mechanisms and/or processes underlying each subtask and inference mentioned in that architecture. The conjecture of our team is that any bottom-up organization may be made operational using two biologically inspired methods called (1) "algorithmic lateral inhibition" (ALI), a generalization of lateral inhibition (LI) anatomical circuits [4] and (2) "accumulative computation" (AC) [19]. Firstly, ALI is considered in the following section and AC in the next one.

\subsection{Algorithmic lateral inhibition}

\subsubsection{Lateral inhibition at the physical level}

There are some neuronal structures that are repeated at all the integration levels in the nervous system and that emerge again in the global behavior of human beings. This leads us to think that they have endured the evolution process because they were adaptively useful for interacting with the environment. If we look at these structures from an electronic and computational perspective we could say that they are the "basic functional modules" in terms of which evolution has designed the best architecture for the nervous system (NS) to process information. This is the case of the lateral inhibition circuits, due to the wide range of functions which they may synthesize, to their capacity of explaining in neurophysiology, and their facility of abstraction when accepting to maintain invariant their structure in front of semantic level changes.

LI schemes may be found in levels such as neurogenesis, dendrodendritic contacts, neuronal circuits in the retina, lateral geniculate body and in cerebral cortex, in the interaction between groups of neurons (ocular and orientation dominance columns) [32]. At the physical level, in terms of interconnected circuits that are described using a language of signals, there are two basic connectivity schemes: (1) non-recursive LI, and (2) recursive LI, with feedback (as outlined in Fig. 1). In non-recursive LI, the modification of a unit response depends on the inputs to the neighboring units, and in recurrent LI it depends on the outputs of the neighboring units.

If extending the formulation to the continuous case (see Fig. 1b), the terms of interaction become nuclei of a convolution integral, so that the output, $\Phi(x, y)$, is the result of accumulating the direct excitation of each unit, $I(x, y)$, with the inhibition from modulating the excitation received by the connected neighboring units, $I(\alpha, \beta)$, via the weight factors $K(x, y ; \alpha, \beta)$. The difference nucleus, $K(x, y ; \alpha, \beta)$, is now responsible for the specific form of the calculation which, in all cases, acts as a detector of contrasts. In the recursive LI, it is the direct output, $\Phi(x, y)$, which accumulates the inhibition from the responses of the neighboring units, $\Phi(\alpha, \beta)$, weighted by an interaction coefficient $K^{*}(x, y ; \alpha, \beta)$, basically different from that of the direct path $(K)$.

Here again, in recursive $L I$, the shape and size of the receptive field (the interaction nucleus $K^{*}$ ) specifies the network connectivity (cooperation-competition area) and the calculation details (syntony, orientations, shapes, speeds, and so on). The most usual shape in $K$ and $K^{*}$ is obtained from subtracting two Gaussians. Thus the calculation structures inherent in the entire LI network are obtained: (1) a central area (ON or OFF), (2) a peripheral area (OFF or ON), and (3) an excluded region (outside the receptive field).

\subsubsection{Algorithmic lateral inhibition at the symbol level}

The LI model at the physical level is limited to a language of physical signals as functions of time. The first possible abstraction, which passes from a circuit to an algorithm, is obtained by rewriting the accumulation and inhibition processes in terms of rules (conditionals "if-then"), as a generalization of the weighted sum and the threshold. The condition field of a rule generalizes the weighted sum and the conditional generalizes the non-linearity of the threshold. Furthermore, the structure of the LI model is maintained. In other words, now also receptive fields (data fields in a FIFO memory) with an excitatory center and an inhibitory periphery, both for the input space (non-recursive LI) and for the output space (recursive IL) are defined. However, the nature of these input and output spaces is changed drastically, to be spaces of representation; that is, they are multidimensional spaces, where each "axis" represents possible values of an independent variable which measure an independent possible property of inputs and outputs. For the input space, the properties are those pertinent to the description of the external and internal "world", as seen by the previous neural nets. For the output space, properties are "decisions" of the net under consideration in the proper time axis. In the simplest case, the axons of each neuron of the net are a

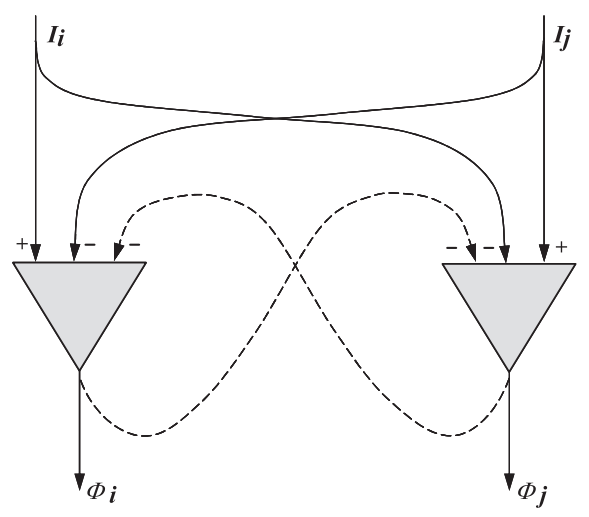

b

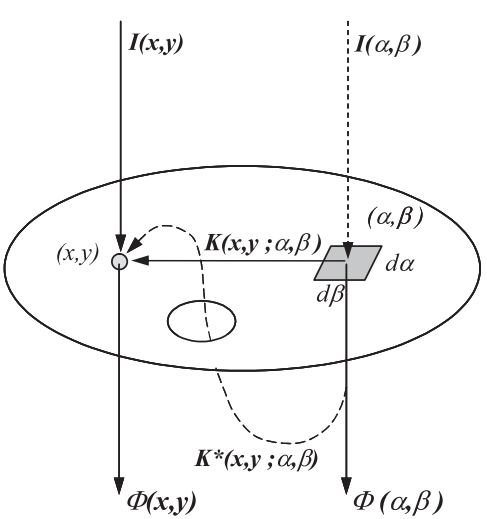

Fig. 1. Non-recursive (continuous line) and recursive (discontinuous line) LI connectivity schemes at the physical level: (a) discrete case and (b) continuous case. 
proposing a sequence of "decisions" that fill in the output FIFO memory. Additionally, the decision rules in ALI need not be limited to analytic but can include also logical-relational operators as components of the calculus carried out by its condition fields [4,32].

With this interpretation of ALI, each element of calculus samples its data in the center and periphery of the volume specified by its receptive field in the input space, and also samples in the center and periphery of the volume which specifies its receptive field in the output space. By specifying the nature of the decision rules the different types of calculus attainable by a network of algorithmic lateral inhibition (ALI) are obtained.

\subsubsection{Algorithmic lateral inhibition at the knowledge level}

To complete the possibilities of the LI network, we do a new abstraction process and we pass from the symbol level to the knowledge level by generalizing the rules in terms of inferences $[5,32]$. Through this abstraction process it is possible to consider ALI circuits as the anatomical support of an inferential scheme (see Fig. 2).

\subsection{Accumulative computation}

Usually the time evolution of the neuron membrane potential is modeled by a first order differential equation known as the "leaky integrator model". If we move from differential equations to equations in finite differences then we get the temporal ALI model previously described, in which each element of calculus samples its input and feedback data from two FIFO memories. A different way of modeling time evolution of membrane potential is consider the membrane as a local working memory in which neither the triggering conditions nor the way in which the potential tries to return to its input-free equilibrium value, needs to be restricted to thresholds and exponential increases and decays. This type of working memory was named "accumulative computation" (AC) $[7,8,33]$, and is characterized by the possibility of controlling its charge and discharge dynamics in terms of:

(1) The presence of specific spatio-temporal features with values over a certain threshold.

(2) The persistency in the presence of these features.

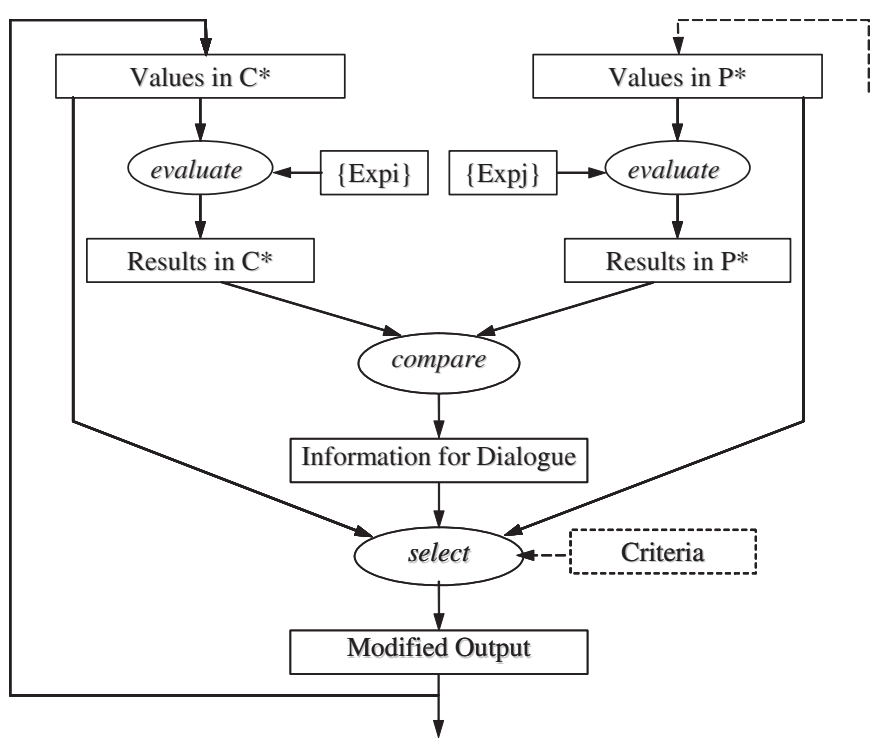

Fig. 2. Recurrent ALI inferential scheme. The results of the evaluation in the central $\left(\mathrm{C}^{*}\right)$ and peripheral $\left(\mathrm{P}^{*}\right)$ parts of the feedback receptive field are first compared and the result of this comparison is used to select the updated output (see [34]).

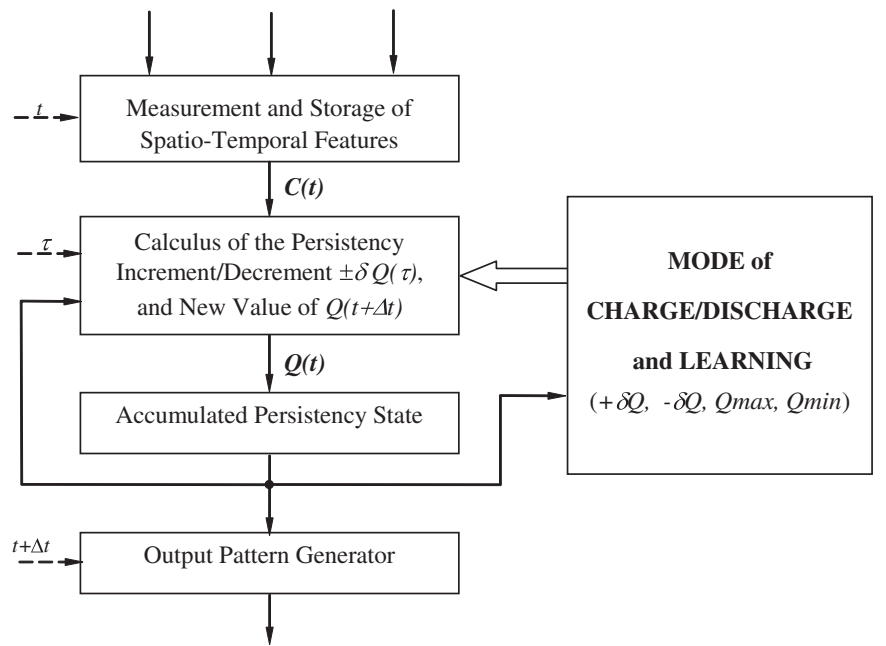

Fig. 3. The AC working memory model.

(3) The increment or decrement values $( \pm \delta Q)$ in the accumulated state of activity of each feature and the corresponding current value, $Q(t)$.

(4) The control (Mode of charge/discharge) and learning mechanisms.

Fig. 3 shows the block diagram of the accumulative computation model. The model works in two time scales, a macroscopic, $t$, associated to the external data sequence to be processed by the net and a microscopic one, $\tau$, associated to the set of internal processes that take place while the external data (an image, for instance) remain constant.The control rules used to calculate the persistency of motion through time are

$Q[x, y ; t+\Delta t]= \begin{cases}\max \left(Q[x, y ; t]-\delta Q, Q_{\min }\right) & \text { if } C[x, y ; t]=1 \\ \min \left(Q[x, y ; t]+\delta Q, Q_{\max }\right) & \text { otherwise }\end{cases}$

where $C[x, y ; t]=1$ means that motion has been detected on pixel $[x, y]$ at $t$, and $Q_{\max }$ and $Q_{\min }$ are the maximum and minimum charge values, respectively. When providing values to $-\delta Q,+\delta Q, Q_{\max }$ and $Q_{\text {min }}$, the different working modalities of the AC are obtained.

In Fig. 4 the behavior of the accumulative computation model is shown in one-dimensional and very easy situations. Let us suppose that values of $I(x, y ; t)$ correspond to an indefinite sequence of images where several objects are moving. Let us also suppose that the measured property, $p(x, y ; t)$ is simply the result of the binary threshold of image $I(x, y ; t)$. Then, the control mode compares values of $p(x, y ; t)$ in two successive instants, interpreting that $p(x, y ; t)=1$ means that there is a moving object over pixel $(x, y)$ at $t$ and that $p(x, y ; t)=0$ means there is no moving object. Thus, changes $p(t-\Delta t)=0$ to $p(t)=1$ mean that a moving object has entered that unit's receptive field (RF). If $p(t-\Delta t)=1$ and $p(t)=0$, a moving object has left the RF; if both are zero, there is no moving object over the RF, and, finally, if both are one, there is a moving object crossing over the RF. For this property, the evolution of its persistency, $Q(t)$, is shown in Fig. 4 for some modalities of use selected.

\section{Applications in computer vision}

In this section we will re-explore the fact that with only two synthetic mechanisms, ALI and AC, it is sufficient to do computational an important part of the visual processes. Some of the works of our group support the validity of this methodological approximation, as described in this part of the paper.

Firstly, AC was applied to the problem of the classification of moving objects in long image sequences [7], calculating the 
a

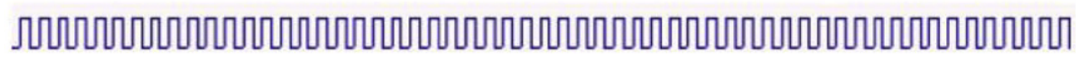

b

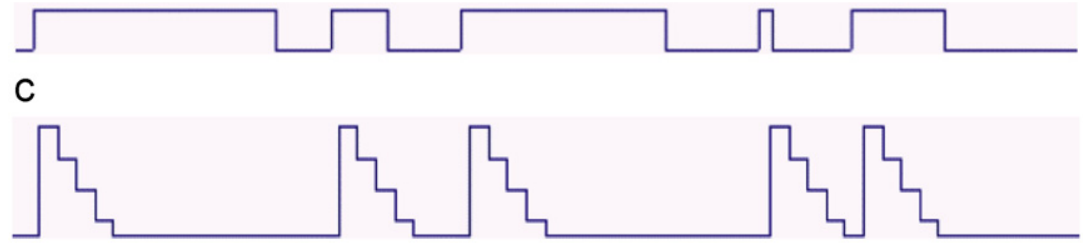

d

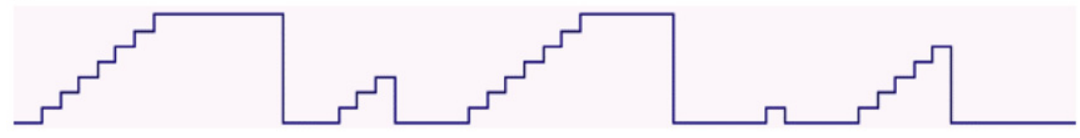

e

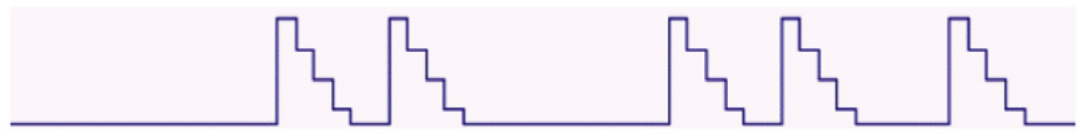

f

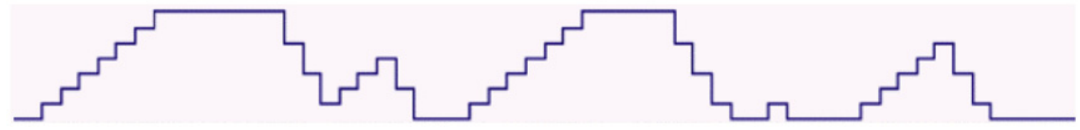

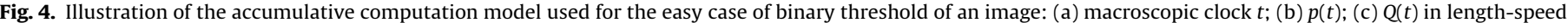
relation modality; (d) $Q(t)$ in input modality; (e) $Q(t)$ in output modality; and (f) $Q(t)$ in charge/discharge modality.

length-speed relation (LSR) starting from Eq. (2), and showing its capacity to be implemented in real-time $[9,18,6]$. AC also was applied successfully to detect and eliminate noise in the segmentation of moving objects in sequences of video [1]:

$Q[x, y ; t+\Delta t]= \begin{cases}\max (Q[x, y ; t]+\delta Q, Q \min ) & \text { if } C[x, y ; t]=1 \\ Q \min & \text { otherwise }\end{cases}$

Later on the combination of AC and ALI (introducing a second time scale into the equation) was used in the resolution of the problem of segmenting moving silhouettes in video sequences $[10,11]$. Its neuronal nature was described in detail [12], as well as the model for motion detection [13] and the influence of each parameter of the combination between AC and ALI [14]. The object segmentation task is composed of subtasks "charge-based pixel charge computation" (using AC and ALI with macroscopic clock), "charge-based blob fusion" (ALI, and microscopic and macroscopic clocks) and "charge-based shape fusion" (ALI and AC, and microscopic and macroscopic clocks).

The novel model based in neural networks combining AC and ALI was denominated "lateral interaction in accumulative computation" (LIAC) [13]. The LIAC structure is built as an artificial neural network (ANN) and hence it inhabits a learning system by itself. Nevertheless, the learning capability of the LIAC method has been extended through the modification of its parameters [31]. This optimization problem used a genetic algorithm (GA) in order to obtain the best sets of parameters for an enhanced segmentation.

From the good results obtained by means of these methods in computer-vision-based motion analysis, the following step was the challenge of facing selective visual attention (dynamic) by means of a research line $[22,23]$ where the importance of the incorporation of new parameters appeared. This research aims in describing a method for visual surveillance based on biologically motivated dynamic visual attention in video image sequences. The system is based on the extraction and integration of local (pixels and spots) as well as global (objects) features. Our approach defines a method for the generation of an active attention focus on a dynamic scene for surveillance purposes. The system segments in accordance with a set of predefined features, including gray level, motion and shape features, giving raise to two classes of objects: vehicle and pedestrian. The solution proposed to the selective visual attention problem consists of de-composing the input images of an indefinite sequence of images into its moving objects, defining which of these elements are of the user's interest at a given moment, and keeping attention on those elements through time. Features extraction and integration are solved precisely by incorporating the mechanisms described, AC and ALI.

An important problem in $\mathrm{AI}$ is to find calculation procedures to save the semantic gap between the analytic formulations of the neuronal models and the concepts of the natural language used to describe the cognitive processes. Therefore, in a recent work [37], we explored a way of saving this gap for the case of the attentional processes.

Among others, we also introduced velocity to improve the capture of the attention on the objects in movement [24]. In this case, we highlight the importance of the motion features present in our algorithms in the task of refining and/or enhancing scene segmentation in the methods proposed. The estimation of these motion parameters is performed at each pixel of the input image by means of the AC method. "Motion presence", "module of the velocity" and "angle of the velocity", all obtained from AC computation method, are shown to be very significant to adjust different scene segmentation outputs in our dynamic visual attention method $[25,26]$.

In parallel with the previous works, again mainly AC was used, and ALI in a minor degree, to improve the segmentation of moving objects, by introducing stereovision, with the purpose of adding a parameter that can contribute enormously in any tracking task in 
the three-dimensional world; this is parameter depth [27]. In this work a method that turns around the existing symbiosis between stereovision and motion is used; motion minimizes correspondence ambiguities, and stereovision enhances motion information. The central idea behind our approach is to transpose the spatially defined problem of disparity estimation into the spatial-temporal domain. Motion is analyzed in the original sequences by means of AC and the disparities are calculated from the resulting twodimensional motion charge maps [29,15,17].

During the last few years the first results applied to the potential usefulness in mobile robotics have also been introduced [28]. Also, some recent work on combining $\mathrm{AC}$ and fuzzification techniques has been presented [38]. Finally, the LI model at the knowledge level has shown to be a good method to solve problems of cooperation and dialogue in the theory of multi-agent systems [34].

To date, the research team goes on working with the two neuronal processes, but now applied to segmentation and tracking in color video sequences. We are delighted to offer some initial results for the BEHAVE Test Case Scenario 0 (downloaded from http://groups.inf.ed.ac.uk/vision/BEHAVEDATA/ INTERACTIONS/). Fig. 5a to f show some frames with significant outputs. In the input video frames red and yellow bounding boxes stand for moving objects as segmented in the RGB and HLS color spaces, respectively. Fig. 5a shows frames 9303, 9320, 9333, 9359 and 9363 containing a moving car and people moving slowly. In Fig. 5b we have frames 3684, 3692, 3704, 3713 and 3728, where you may observe a moving cyclist and some stationary people. Fig. $5 \mathrm{c}$ and $\mathrm{d}$ are examples of the presence of moving people (frames $6327,6357,6369,6376$ and 6398, as well as frames 102-104, 107 and 109). Fig. 5e, composed of frames $122-125$ and 133, contains people with little motion. Lastly, we offer the results of the segmentation of a single moving human in Fig. $5 \mathrm{f}$ (frames 4379-4383).

\section{Conclusions}

Neurally inspired computer vision involves at least memory processes, alert, surveillance, selective attention, divided attention, reinforcement learning, intentionality and conscience. The complexity of these mechanisms is the reason for the difficulty found when trying to synthesize them computationally. A satisfactory solution, close to biology, is still a long term objective.

In this work we have revisited the works in computer vision by using a description framework based in the use of two basic neuronal mechanisms, the lateral inhibition and the working

a

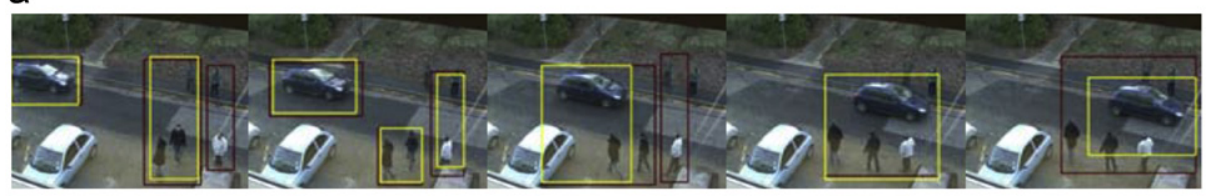

b

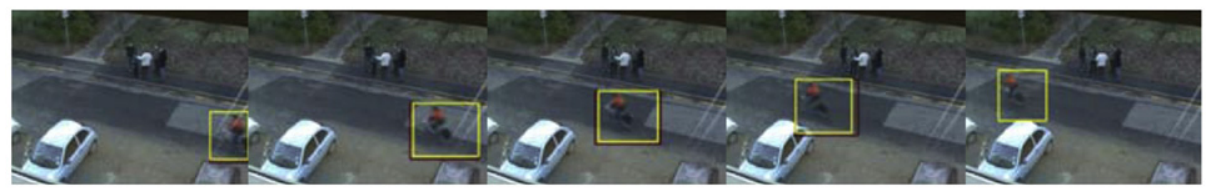

C

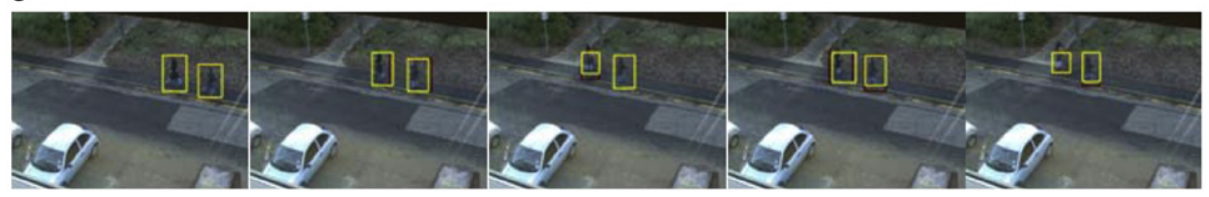

d

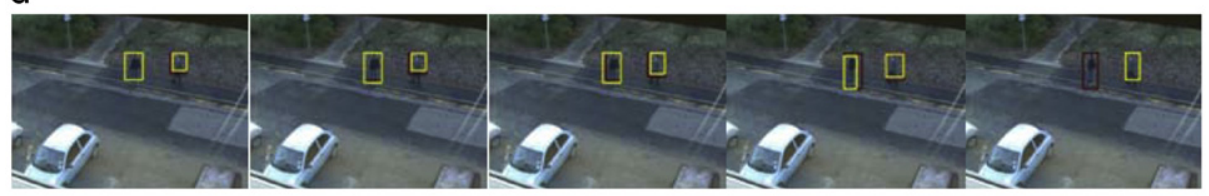

e

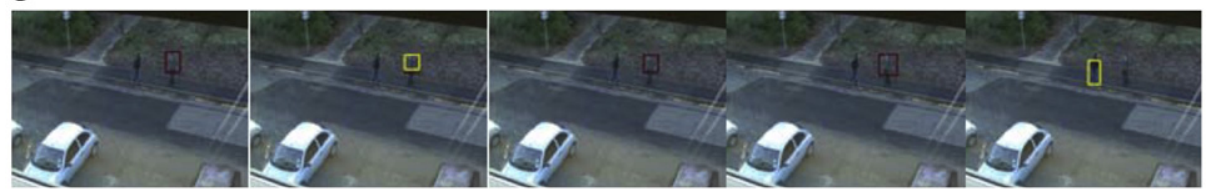

f

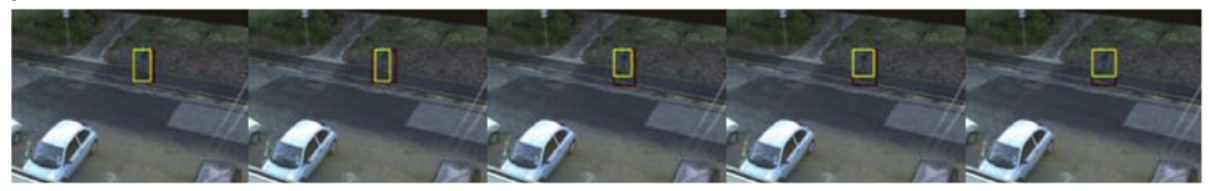

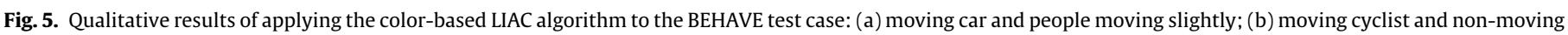
people; (c and d) moving people; (e) people moving slowly; and (f) a moving human. 
memory related to the temporal evolution of the membrane potential, which was denominated accumulative computation. In order to extract the maximum performance the two mechanisms at the three levels used in Artificial Intelligence to describe a calculation (the physical level, the level of the symbols and the knowledge level) have been formulated. We have proposed a method to interpret the function performed by a neural circuit, not only at the level of neurophysiologic signals but also in terms of symbolic rules and inferential schemes.

In the previous section, we have also commented some of the results obtained by the research team from using the conceptual scheme described in the previous sections. Concretely, AC has been applied to the problem of the classification of moving objects in image sequences. The combination of AC and ALI has been used in the resolution of the problem of segmenting moving silhouettes in video sequences. A model based in neural networks combining AC and ALI, denominated "lateral interaction in accumulative computation" (LIAC) has been created. The selective visual attention (dynamic) has been faced by these methods describing a method for visual surveillance applied to video image sequences. For the case of the attentional processes, we also have explored a way of saving the semantic gap between the analytic formulations of the neuronal models and the concepts of the natural language used to describe the cognitive processes. We also have introduced mechanisms to improve the capture velocity of the objects in movement, refining and/or enhancing scene segmentation in the methods proposed. In a minor degree, AC and ALI have been used to improve the segmentation of moving objects by introducing stereovision, with the purpose of improving any tracking task in the threedimensional world. During the last few years, the first results applied to the potential usefulness in mobile robotics have also been introduced. To date, the research team goes on working with the two neuronal processes, but now applied to segmentation and tracking in color video sequences.

All the above results, although reasonable in AI, are far away from approaching biological solutions for at least two reasons. The first one is the enormous constituent difference between biological systems and synthesis elements used in computer vision. The second reason is the ignorance on the architectures of cognition, starting from the doubt on the suitability of the term architecture to describe perception in humans.

Nevertheless, when seeking to construct solutions able to be computed with programs, cameras and robots, there is no other way than trying to equip the knowledge that we have on the biological solution to the problem of attention with structure and organization. Anything that we cannot describe in a structured, declarative, clear, precise and formal way is not computable. That is why we think that our approach of firstly looking into Neuroscience as an inspiration source, then proposing real computational models, and at the same time evaluating its efficiency in problem solving, independently of its biological origin, is useful.

\section{Acknowledgements}

This work was partially supported by the Spanish Ministerio de Ciencia e Innovación under Projects TIN2007-67586-C02 and TIN2010-20845-C03, and by the Spanish Junta de Comunidades de Castilla-La Mancha under Projects PII2I09-0069-0994, PII2I090071-3947 and PEII09-0054-9581.

\section{References}

[1] E.J. Carmona, J. Martínez-Cantos, J. Mira, A new video segmentation method of mobile objects based on blob-level knowledge, Pattern Recognition Letters 29 (3) (2008) 272-285.
[2] G. Deco, E.T. Rolls, A neurodynamical cortical model of visual attention and invariant object recognition, Vision Research 44 (6) (2004) 621-642.

[3] G. Deco, E.T. Rolls, Attention, short-term memory, and action selection: a unifying theory, Progress in Neurobiology 76 (4) (2005) 236-256.

[4] A.E. Delgado, J. Mira, R. Moreno-Díaz, A neurocybernetic model of modal cooperative decision in the Kilmer-McCulloch space, Kybernetes 18 (3) (1989) $48-57$

[5] A.E. Delgado, J. Mira, Algorithmic lateral inhibition as a generic method for visual information processing with potential applications in robotics, Computational Intelligent Systems for Applied Research (2002) 477-484.

[6] A.E. Delgado, M.T. López, A. Fernández-Caballero, Real-time motion detection by lateral inhibition in accumulative computation, Engineering Applications of Artificial Intelligence 23 (1) (2010) 129-139.

[7] M.A. Fernández, Una arquitectura modular de inspiración biológica con capacidad de aprendizaje para el análisis de movimiento en secuencias de imagen en tiempo real, Ph.D. Thesis, UNED, Madrid, Spain, 1995.

[8] M.A. Fernández, J. Mira, M.T. López, J.R. Alvarez, A. Manjarrés, S. Barro, Local accumulation of persistent activity at synaptic level: application to motion analysis, Lecture Notes in Computer Science, vol. 930, 1995, pp. 137-143.

[9] M.A. Fernández, A. Fernández-Caballero, M.T. López, J. Mira, Length-speed ratio (LSR) as a characteristic for moving elements real-time classification, RealTime Imaging 9 (1) (2003) 49-59.

[10] A. Fernández-Caballero, Modelos de interacción lateral en computación acumulativa para la obtención de siluetas, Ph.D. Thesis, UNED, Madrid, Spain, 2001

11] A. Fernández-Caballero, J. Mira, M.A. Fernández, M.T. López, Segmentation from motion of non-rigid objects by neuronal lateral interaction, Pattern Recognition Letters 22 (14) (2001) 1517-1524.

[12] A. Fernández-Caballero, J. Mira, M.A. Fernández, A.E. Delgado, On motion detection through a multi-layer neural network architecture, Neural Networks 16 (2) (2003) 205-222.

[13] A. Fernández-Caballero, J. Mira, A.E. Delgado, M.A. Fernández, Lateral interaction in accumulative computation: a model for motion detection, Neurocomputing 50 (2003) 341-364.

[14] A. Fernández-Caballero, M.A. Fernández, J. Mira, A.E. Delgado, Spatio-temporal shape building from image sequences using lateral interaction in accumulative computation, Pattern Recognition 36 (5) (2003) 1131-1142.

[15] A. Fernández-Caballero, M.T. López, J. Mira, A.E. Delgado, J.M. López-Valles M.A. Fernández, Modelling the stereovision-correspondence-analysis task by lateral inhibition in accumulative computation problem-solving method, Expert Systems with Applications 33 (4) (2007) 955-967.

[16] A. Fernández-Caballero, J. Mira, G. Deco, 50 years of artificial intelligence: a neuronal approach, Neurocomputing 71 (4-6) (2008) 667-669.

[17] A. Fernández-Caballero, M.T. López, S. Saiz-Valverde, Dynamic stereoscopic selective visual attention DSSVA: integrating motion and shape with depth in video segmentation, Expert Systems with Applications 34 (2) (2008) 1394-1402.

[18] A. Fernández-Caballero, M.T. López, J.C. Castillo, S. Maldonado-Bascón, Realtime accumulative computation motion detectors, Sensors 9 (12) (2009) 10044-10065.

[19] A. Fernández-Caballero, M.T. López, M.A. Fernández, J.M. López-Valles, Revisiting algorithmic lateral inhibition and accumulative computation, Lecture Notes in Computer Science, vol. 5601, 2009, pp. 57-66.

20] D. Heinke, G.W. Humphreys, Computational models of visual selective attention: a review, Connectionist Models in Cognitive Psychology 1 (4) (2005) 273-312.

[21] D. Heinke, G.W. Humphreys, C.L. Tweed, Top-down guidance of visual search: a computational account, Visual Cognition 14 (4-8) (2006) 985-1005.

[22] M.T. López, Modelado computacional de los mecanismos de atención selectiva mediante redes de interacción lateral, Ph.D. Thesis, UNED, Madrid, Spain, 2004.

[23] M.T. López, A. Fernández-Caballero, J. Mira, A.E. Delgado, M.A. Fernández Algorithmic lateral inhibition method in dynamic and selective visual attention task: application to moving objects detection and labeling. Expert Systems with Applications 31 (3) (2006) 570-594.

[24] M.T. López, A. Fernández-Caballero, M.A. Fernández, J. Mira, A.E. Delgado Motion features to enhance scene segmentation in active visual attention, Pattern Recognition Letters 27 (5) (2006) 469-478.

[25] M.T. López, A. Fernández-Caballero, M.A. Fernández, J. Mira, A.E. Delgado Visual surveillance by dynamic visual attention method, Pattern Recognition 39 (11) (2006) 2194-2211.

[26] M.T. López, A. Fernández-Caballero, M.A. Fernández, J. Mira, A.E. Delgado Dynamic visual attention model in image sequences, Image and Vision Computing 25 (5) (2007) 597-613.

[27] J.M. López-Valles, Estereopsis y movimiento. Modelo de disparidad de carga: Un enfoque con inspiración biológica, Ph.D. Thesis, Universidad de Castilla-La Mancha, Albacete, Spain, 2004.

[28] J.M. López-Valles, M.A. Fernández, A. Fernández-Caballero, M.T. López J. Mira, A.E. Delgado, Motion-based stereovision model with potential utility in robot navigation, Lecture Notes in Artificial Intelligence, vol. 3533, 2005 pp. $16-25$.

[29] J.M. López-Valles, M.A. Fernández, A. Fernández-Caballero, Stereovision depth analysis by two-dimensional motion charge memories, Pattern Recognition Letters 28 (1) (2007) 20-30.

[30] D. Marr, Vision. A Computational Investigation into the Human Representation and Processing of Visual Information, W.H. Freeman and Company, 1982. 
[31] J. Martínez-Cantos, E.J. Carmona, A. Fernández-Caballero, M.T. López, Parametric improvement of lateral interaction in accumulative computation in motion-based segmentation, Neurocomputing 71 (4-6) (2008) 776-786.

[32] J. Mira, A.E. Delgado, What can we compute with lateral inhibition circuits? Lecture Notes in Computer Science, vol. 2084, 2001, pp. 38-46.

[33] J. Mira, M.A. Fernández, M.T. López, A.E. Delgado, A. Fernández-Caballero, A model of neural inspiration for local accumulative computation, Lecture Notes in Computer Science, vol. 2809, 2003, pp. 427-435.

[34] J. Mira, A.E. Delgado, A. Fernández-Caballero, M.A. Fernández, Knowledge modelling for the motion detection task: the algorithmic lateral inhibition method, Expert Systems with Applications 27 (2) (2004) 169-185.

[35] J. Mira, A.E. Delgado, On how the computational paradigm can help us to model and interpret the neural function, Natural Computing 6 (3) (2006) 207-209.

[36] J. Mira, Symbols versus connections: 50 years of artificial intelligence, Neurocomputing 71 (4-6) (2008) 671-680.

[37] J. Mira, A.E. Delgado, M.T. López, A. Fernández-Caballero, M.A. Fernández, A conceptual frame with two neural mechanisms to model selective visual attention processes, Neurocomputing 71 (4-6) (2008) 704-720.

[38] J. Moreno-Garcia, L. Rodriguez-Benitez, A. Fernández-Caballero, M.T. López, Video sequence motion tracking by fuzzification techniques, Applied Soft Computing 10 (1) (2010) 318-331.

[39] A. Newell, The knowledge level, AI Magazine Summer (1981) 1-20.

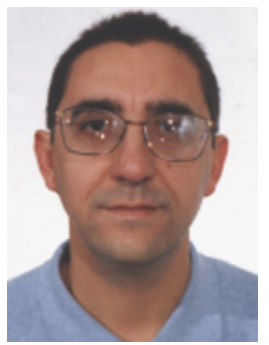

Antonio Fernández-Caballero received his degree in Computer Science from the Technical University of Madrid, Spain, in 1993, and received his Ph.D. from the Department of Artificial Intelligence of the National University for Distance Education, Spain, in 2001. Since 1995, he is an Associate Professor with the Department of Computer Science at the University of Castilla-La Mancha, Spain. His research interests are in Image Processing, Cognitive Vision, Neural Networks, and Intelligent Agents. A. Fernández-Caballero is an Associate Editor of the Pattern Recognition Letters journal and a member of the IAPR. He has authored more than 150 peer reviewed papers.

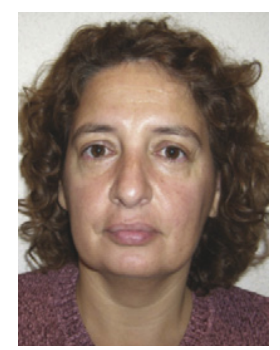

María T. López received her degree in Physics from the University of Valencia, Spain, in 1991, and received her Ph.D. from the Department of Artificial Intelligence of the National University for Distance Education, Spain, in 2004. Since 1991, she is an Associate Professor with the Department of Computing Systems at the University of Castilla-La Mancha, Spain. Her research interests are in Image Processing and Computer Vision. María T. López is member of the IAPR.

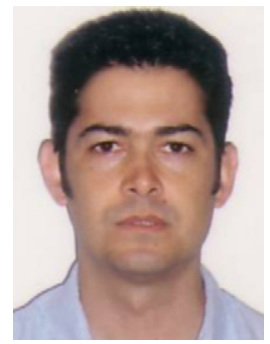

Enrique J. Carmona received his degree in Electronic Engineering from the University of Granada, Spain, in 1996, and received his Ph.D. in Physics from the National University for Distance Education (UNED) in Madrid (Spain), in 2003. Since 2009, he is an Associate Professor in the Department of Artificial Intelligence at the UNED, Spain. His research interests are related to Machine Learning, Computer Vision and Evolutionary Computation.

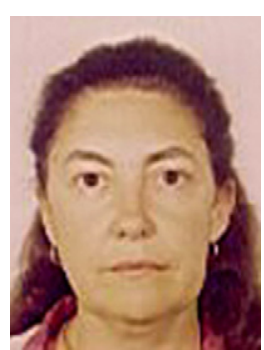

Ana E. Delgado is a Professor of Computer Science and Artificial Intelligence with the Department of Artificial Intelligence at the National University for Distance Education (UNED) in Madrid (Spain). Her current research interests are in Neural Modeling, Bio-inspired Cooperative Agents and Computer Vision. 